\title{
SEGNALAZIONI FAUNisTiCHE ITALIANE
}

\section{9 - Scytodes univittata Simon, 1882 (Araneae, Scytodidae)}

BRESCOVIT A.D. \& Rheims C.A., 2000 - On the synanthropic species of the genus Scytodes Latreille (Araneae, Scytodidae) of Brazil, with synonymies and records of these species in other Neotropical countries. Bulletin of the British Arachnological Society, 11: 320-330.

ÖZkÜtüK R.S., Marusik Y.M., Danişman T., Kunt K.B., YaĞMur E.A. \& Elverici M., 2013 - Genus Scytodes Latreille, 1804 in Turkey (Araneae, Scytodidae). Hacettepe Journal of Biology and Chemistry, 41: 9-20.

Prima segnalazione per l'Italia e seconda segnalazione per l'Europa di specie sinantropa ad ampia distribuzione.

REPERTI. Liguria: Quarto dei Mille (Genova), interno di abitazione privata, 10 m, 4.IX.2015, 1 q, C. Giusto leg., (A. Trotta det. \& coll.); Lazio, Roma, Hotel Canova, 13.V.2010, 1 ô, A. Hänggi leg. (A. Hänggi det., coll. Natural History Museum Basel); Lazio, Roma, abitazione privata, 2013, 1 §̊, 1 ค, G. Tropea leg. (P. Pantini det., coll. Museo Scienze naturali di Bergamo).

OsSERVAZIONI. Specie per lo più sinantropa, diffusa in Centro-Sud America, Isole Canarie, Spagna, Somalia, Seychelles, Yemen, Iran, Turkmenistan, Kirghizistan, India e Myanmar.

Alessio TROTTA via delle Ginestre 1/A2, 17024 Finale Ligure (SV), Italia. E-mail: alessiotrotta1973@libero.it

\section{0 - Dysgonia torrida (Guenée, 1852) (Lepidoptera, Erebidae, Erebinae)}

Goater B., ROnKay L., Fibiger M., 2003 - Catocalinae \& Plusiinae. Noctuidae Europaeae, 10.

Prima segnalazione per il Piemonte di specie paleotropicale-subtropicale migratrice, già citata sporadicamente di poche altre regioni italiane.

RePERTI. Piemonte: Langhe, Pezzolo Valle Uzzone (CN), 321 m, 16.IX.2015, 1 ô, E. Gallo leg. (coll. Gallo, Genova). Identificazione confermata mediante l'esame dell'apparato copulatore (Prep. gen. GAL 892).

OsSERVAZIONI. Ampiamente diffusa in tutta l'Africa e dal Vicino Oriente al Sud-Est asiatico, D. torrida è invece rara in Europa, dove è stata rinvenuta solo nei settori più meridionali del Continente, dalla Penisola iberica a quella balcanica, prevalentemente in località costiere. Ritenuta estranea alla nostra fauna da Berio (1991), la sua presenza in Italia, già segnalata da Zilli (1990), ha ricevuto in seguito ulteriori conferme. In base alle attuali conoscenze (Bertaccini et al., 2008), le segnalazioni più numerose sono quelle relative alla Sicilia, mentre poche altre si riferiscono a Calabria, Basilicata, Puglia, Lazio e Sardegna. Più recentemente la specie è stata rinvenuta anche in Umbria (Zerunian \& Zilli, 2014). Per quanto invece concerne l'Italia settentrionale, il numero dei reperti è veramente esiguo: sono note infatti soltanto alcune catture effettuate in Veneto e una in Romagna. Una tale scarsità di dati per questa specie in Italia potrebbe comunque dipendere, oltre che dalla sua effettiva rarità, anche da una sottostima della sua presenza dovuta all'estrema somiglianza esterna con la congenere $D$. algira (Linnaeus, 1767), più o meno diffusa in tutta la nostra Penisola, con la quale potrebbe essere stata confusa. Sarebbe pertanto opportuno che gli esemplari conservati nelle collezioni pubbliche e private, determinati come $D$. algira, venissero attentamente controllati. A tal proposito vale la pena segnalare un recente lavoro di Mazel \& Pérez-De Gregorio (2015), i quali hanno sensibilmente incrementato i dati relativi alla presenza di $D$. torrida in Francia meridionale e Spagna proprio esaminando i genitali di esemplari di collezione precedentemente identificati come $D$. algira. La cattura effettuata a Pezzolo V. U., dove è presente anche D. algira, è avvenuta di sera alla luce di un lampione.

BIBLIOGRAFIA

BerIo E., 1991 - Lepidoptera Noctuidae. II. Sezione Quadrifide - Fauna d'Italia, Vol. XXVII. Calderini, Bologna. 708 pp.

Bertaccini E., Fiumi G., Parenzan P., Zilli A., 2008 - Lepidotteri Eteroceri d'Italia. Noctuidae, Vol. I, Calpinae-Catocalinae, Natura Edizioni Scientifiche, Bologna. 288 pp.

Mazel R., Pérez-De Gregorio J.J., 2015 - Dysgonia torrida (Guenée, 1852), espèce à «décrypter» en France et en Espagne. Revue de l'Association Roussillonnaise d'Entomologie, 24: 197-199. 
Zerunian Z., Zilli A., 2014 - The Macrolepidoptera of Mt Subasio (Umbria, Central Italy), pp. 7-119. In: ZiLli A. (ed.), Lepidoptera research in areas with high biodiversity potential in Italy. Vol. 1. Natura Edizioni Scientifiche, Bologna.

ZILLI A., 1990 - Novità faunistiche e zoogeografiche sui Nottuidi italiani (Lepidoptera, Noctuidae). Bollettino dell'Associazione Romana di Entomologia, 44: 33-53.

Enrico GALLO

Via Trento 5/20D, 16145 Genova, Italia. E-mail: engallo1936@libero.it

611 - Choragus sheppardi Kirby, 1819 (Coleoptera, Anthribidae, Choraginae)

AbBazzi P., Bartolozzi L., CAlamandrei S., 1999 - Contributo alla conoscenza degli Anthribidae italiani (Insecta, Coleoptera, Curculionoidea). Annali del Museo Civico di Storia Naturale G. Doria, 93: 57-106

Prima segnalazione della specie per l'Appennino tosco-romagnolo e per il Parco Nazionale delle Foreste Casentinesi, Monte Falterona e Campigna.

RePerti. Parco Nazionale delle Foreste Casentinesi, Monte Falterona e Campigna: Toscana, provincia di Firenze, comune di San Godenzo, Poggio Citerna, circa 1000 m s.l.m., 29.VII.2016, M. Norbiato \& L. Bartolozzi leg., 5 exx., su Fagus sylvatica L. (Museo di Storia Naturale dell’Università di Firenze, Sezione di Zoologia; $\mathrm{n}^{\circ}$ collezione 18101).

OsSERVAZIONI. Specie diffusa nella maggior parte dell'Europa e in Asia Minore (Frieser, 1981a; 1981b; Trýzna \& Valentine, 2011). In Italia nota di Liguria, Piemonte, Lombardia, Trentino-Alto Adige, Lazio, Sardegna (Abbazzi \& Osella, 1992; Colonnelli \& Sacco, 1997), nonché di Friuli-Venezia Giulia, Emilia-Romagna e Toscana (Abbazzi et al., 1999). Per la Toscana era segnalato delle province di Arezzo, Grosseto e Firenze; per quest'ultima tuttavia in zona di relativamente bassa quota (comune di Figline Valdarno, dintorni di Gaville, circa $400 \mathrm{~m}$ ). Questo ritrovamento rappresenta, quindi, la prima segnalazione per l'Appenino tosco-romagnolo. Non era stata precedentemente segnalata nel lavoro dedicato ai coleotteri xilofagi e subcorticicoli del Parco Nazionale delle Foreste Casentinesi, Monte Falterona e Campigna (Cecchi \& Bartolozzi, 1997) e quindi il presente ritrovamento rappresenta anche il primo per quest'area protetta. I nuovi reperti del comune di San Godenzo sono stati raccolti di giorno, battendo un sottile tronco morto di faggio (Fagus sylvatica L.), mediante ombrello entomologico. Da notare che, oltre ad essere molto piccoli e non sempre immediatamente riconoscibili come antribidi, questi coleotteri saltano via molto rapidamente quando si sentono minacciati e quindi la loro raccolta non è sempre agevole. Nello stesso tronco sono stati osservati anche esemplari di altri coleotteri xilofagi appartenenti alle famiglie Colydiidae, Scolytidae e Latridiidae. L'habitat circostante era costituito da bosco di faggio situato lungo un crinale, con la presenza di molti alberi morti in piedi o deperienti. Choragus sheppardi è un coleottero la cui larva si sviluppa nel legno morto, su rami infestati da funghi corticicoli (Diatrypaceae) come Sphaeria stigma Hoffm. (Abbazzi et al., 1999; Olberg et al., 2015). La sua presenza è stata riscontrata in diverse latifoglie (castagno, faggio, melo, pero, biancospino, ecc.). Nel nord Europa la specie è stata trovata anche su olmo montano (Ulmus glabra Huds.) in Svezia e su edera (Hedera helix L.) in Gran Bretagna (Olberg et al., 2015). Nella recentissima Lista Rossa dei Coleotteri saproxilici italiani (Carpaneto et al., 2015) la specie è indicata come LC (Least Concern). Da notare come la nomenclatura e la sistematica degli antribidi paleartici siano state parzialmente rimaneggiate da Trýzna \& Valentine (2011). Con questa segnalazione la specie si aggiunge agli altri sei taxa di Anthribidae finora segnalati per il Parco Nazionale: Anthribus nebulosus Forster, 1770, Dissoleucas niveirostris (Fabricius, 1798), Enedreytes hilaris Fåhraeus, 1839, Phaenotherium fasciculatum fasciculatum Reitter, 1891, Platyrhinus resinosus (Scopoli, 1763), Ulorhinus bilineatus (Germar, 1819) (Cecchi \& Bartolozzi, 1997; Abbazzi et al., 2004; Ceccolini \& Norbiato, 2015).

\section{BIBLIOGRAFIA}

Abbazzi P., Bartolozzi L., Crudele G., Sforzi A., 2004 - I Coleotteri Curculionoidea del Parco Nazionale delle Foreste Casentinesi, Monte Falterona e Campigna (Insecta Coleoptera): $1^{\circ}$ contributo. Redia, 86: 81-95.

AbBazzi P., Osella G., 1992 - Elenco sistematico-faunistico degli Anthribidae, Rhinomaceridae. Attelabidae, Apionidae, Brentidae, Curculionidae italiani (Insecta, Coleoptera, Curculionoidea). $1^{\circ}$ parte. Redia, 75: 267-414.

Carpaneto G.M., Baviera C., Biscaccianti A.B., Brandmayr P., Mazzei A., Mason F., Battistoni A., Teofili C., Rondinini 
C., Fattorini S., Audisio P. (eds) - A Red List of Italian Saproxylic Beetles: taxonomic overview, ecological features and conservation issues (Coleoptera). Fragmenta entomologica, 47: 53-126.

Cecchi B., Bartolozzi L., 1997 - I Coleotteri xilofagi e subcorticicoli del Parco Nazionale delle Foreste Casentinesi, Monte Falterona e Campigna (Insecta Coleoptera). Bollettino della Società Entomologica Italiana, 129: 119-139.

Ceccolini F., Norbiato M., 2015 - Contributo alla conoscenza della coleotterofauna della "Foresta della Lama" nel Parco Nazionale delle Foreste Casentinesi, Monte Falterona e Campigna (Insecta Coleoptera Rhysodidae, Lucanidae, Scarabaeidae, Elateridae, Endomichydae, Coccinellidae, Cleridae, Salpingidae, Cerambycidae, Anthribidae, Attelabidae). Quaderno di Studi e Notizie di Storia Naturale della Romagna, 42: 113-136.

Colonnelli E., Sacco F., 1997 - Coleoptera Curculionidea. In: Zapparoli M. (ed.). Gli insetti di Roma. Comune di Roma, Dipartimento X, Area Risorsa Suolo e Tutela Ambiente, Quaderni dell'Ambiente, 6. pp. 217-235.

FRIESER R., 1981a - Die Anthribiden der Westpaläarktis einschließlicb der Arten der UdSSR (Coleoptera, Anthribidae). Mitteilungen der Münchner Entomologischen Gesellschaft, München, 71: 33-107.

Frieser R., 1981b - 90. Familie: Anthribidae (Breitmaulrüßler) (pp. 22-31). In: Freude H., Harde K.W., LohSE G.A. (eds.). Die Käfer Mitteleuropas, 10. Goecke \& Evers, Krefeld, pp.310.

Olberg S., Laugsand A.E., Solev̊̊g P.K., 2015 - The genus Choragus Kirby, 1819 (Coleoptera, Anthribidae) in Norway. Norwegian Journal of Entomology, 62: 129-132.

TrÝzna M., Valentine B.D., 2011 - Anthribidae (pp. 64-66, 90-109). In: Löbl I. \& Smetana A. (eds.). Catalogue of Palaearctic Coleoptera, Vol. 7. Apollo Books, Stenstrup, pp. 373.

Margherita NORBIATO

Reparto di Entomologia, Sezione di Zoologia, Museo di Storia Naturale dell’Università di Firenze

Via Romana 17, Firenze, Italia. E-mail: margherita.norbiato@libero.it

\section{2 - Scaphoideus dellagiustinai Webb \& Viraktamath, 2007 (Hemiptera Cicadellidae)}

DMitriev D.A., 2003 onward - Web site: $3 i$ interactive keys and taxonomic databases. http://dmitriev.speciesfile.org Prima segnalazione per l'Italia di specie diffusa in Francia e Burkina Faso.

RePerti. Toscana: prov. Pisa, Parco di San Rossore, 0 m slm (4343’25.3”N 10¹8’46.4”E), 1 ô, 27.VII.1999 03.VIII.1999 Mazzoni legit, ex trappola Malaise (Mazzoni e Trivellone det., Mazzoni coll.).

OSSERVAZIONI. L'esemplare è stato raccolto in una trappola Malaise collocata nel Parco Naturale di San Rossore nell'estate del 1999, in una pineta di Pinus pinea a circa $2.5 \mathrm{~km}$ dalla costa sabbiosa, caratterizzata da ambienti dunali. Scaphoideus dellagiustinai Webb \& Viraktamath e Scaphoideus titanus Ball sono le uniche due specie di questo genere ad essere state segnalate sul suolo europeo. Il genere Scaphoideus è di origine Neartica sebbene $S$. dellagiustinai sia stato descritto a partire da due esemplari raccolti nel 2003 in Francia nei pressi di Montpellier (Nusillard, 2007). La specie, peraltro, era stata identificata erroneamente come Scaphoideus rubroguttatus Matsumura (Webb e Viraktamath, 2007). La presente segnalazione fa pertanto anticipare di quattro anni la prima raccolta di questa specie in Europa in un sito, quello di San Rossore, che ricorda per molti aspetti la località tipica francese per la presenza di pineta alle spalle delle dune costiere.

BIBLIOGRAFIA

Nusillard B., 2007 - Scaphoideus rubroguttatus Matsumura, 1914, nouveau pour la faune de France (Hemiptera, Auchenorrhyncha). Bulletin de la Société entomologique de France, 112: 131-133.

WebB M.D., ViraKtamath C.A., 2007 - Three new Old World species of the leafhopper genus Scaphoideus Uhler (Hemiptera: Auchenorrhyncha: Cicadellidae). Zootaxa, 1457: 49-55.

Valerio MAZZONI

Research and Innovation Centre, Fondazione Edmund Mach, via E. Mach 1, 38010 S. Michele all'Adige (TN), Italy. E-mail: valerio.mazzoni@fmach.it

Valeria TRIVELLONE

Swiss Federal Research Institute WSL, Biodiversity and Conservation Biology, Zürcherstrasse 111, CH-8903 Birmensdorf, Switzerland.

ANDREA LUCCHI

DiSAAA-a, Università di Pisa, via del Borghetto 80, 56124 Pisa, Italy 


\section{3 - Tegenaria faniapollinis Brignoli, 1978 (Araneae Agelenidae)}

BRIGNOLI P.M., 1978 - Ragni di Turchia IV. Leptonetidae, Dysderidae ed Agelenidae nuovi o interessanti di grotte della Turchia meridionale (Araneae). Quaderni di Speleologia, Circolo Speleologico Romano, 3: 37-54.

Prima segnalazione per l'Italia di una specie nota in Grecia, Turchia e Repubblica di Macedonia.

Reperti. Sicilia, Catania, Grotta Petralia (1205SICT), 19.II.2017, leg. G. Nicolosi, 2 q (Coll. M. Isaia, Dip. di Scienze della Vita e Biologia dei Sistemi, Università di Torino).

OsSERVAZIONI. Specie descritta da Brignoli (1978) su materiale di Turchia (grotta di Harbiye), successivamente rinvenuta in Grecia (Grotta di Maronia; Deltshev, 2008, sub T. paragamiani Deltshev) e Repubblica di Macedonia (Grotta Mechkina Dupka; Deltshev, 2013 sub T. paragamiani Deltshev). Si tratta di una specie moderatamente troglofila non specializzata che, in grotta, trova condizioni favorevoli alla sopravvivenza. Gli esemplari della nuova località italiana sono stati raccolti a vista a pochi metri dall'ingresso della Grotta Petralia, all'interno della zona fotica. La grotta, che ha uno sviluppo planimetrico di oltre 500 metri, è una cavità di scorrimento lavico localizzata nella città di Catania, a 138 m s.l.m.

Il presente contributo estende all'Italia la distribuzione di questa specie, considerata fino ad ora a diffusione balcanica, e rappresenta di fatto la prima segnalazione per il territorio nazionale italiano.

BIBLIOGRAFIA

Deltshev C., 2008 - Two new spider species, Malthonica bozhkovi sp. nov. and Tegenaria paragamiani sp. nov. from Rhodopy Mountains (Bulgaria and Greece) (Araneae: Agelenidae). Zootaxa, 1872: 37-44.

Deltshev C., Komnenov M., Blagoev G., Georgiev T., Lazarov S., Stojkoska E. \& Naumova M., 2013 - Faunistic Diversity of Spiders (Araneae) in Galichitsa Mountain (FYR Macedonia). Biodiversity Data Journal, 1: e977.

Giuseppe NICOLOSI

Centro Speleologico Etneo, Via Valdisavoia 3, 95123 Catania, Italy. E-mail: gnicolosi@hotmail.it

Laboratorio di Ecologia - Ecosistemi Terrestri, Dipartimento di Scienze della Vita e Biologia dei Sistemi, Università di Torino, Via Accademia Albertina 13, 10123 Torino, Italy. E-mail: marco.isaia@unito.it 